Musées, Patrimoine et Culture scientifiques et techniques

$170 \mid 2017$

mars-avril 2017

\title{
Tester l'ergonomie de dispositifs numériques en langue des signes française
}

Gaëlle Lesaffre et Guillaume Ducongé

\section{OpenEdition \\ Journals}

Édition électronique

URL : http://journals.openedition.org/ocim/1751

DOI : 10.4000/ocim. 1751

ISSN : 2108-646X

Éditeur

OCIM

Édition imprimée

Date de publication : 1 mars 2017

Pagination : 11-17

ISSN : 0994-1908

Référence électronique

Gaëlle Lesaffre et Guillaume Ducongé, «Tester l'ergonomie de dispositifs numériques en langue des signes française », La Lettre de I'OCIM [En ligne], 170 | 2017, mis en ligne le 01 mars 2018, consulté le 01 mai 2019. URL : http://journals.openedition.org/ocim/1751 ; DOI : 10.4000/ocim.1751

Ce document a été généré automatiquement le 1 mai 2019.

Tous droits réservés 


\title{
Tester l'ergonomie de dispositifs numériques en langue des signes française
}

\author{
Gaëlle Lesaffre et Guillaume Ducongé
}

Capture d'écran du commentaire introductif du visioguide du musée national Picasso-Paris Audiovisit. À droite, Pablo Picasso, Autoportrait torse nu en culotte de boxeur devant Homme assis au verre en cours d'exécution dans l'atelier de la rue Schoelcher, Paris, 1915-1916. Collection Dora Maar, 1998, MP1998-134.
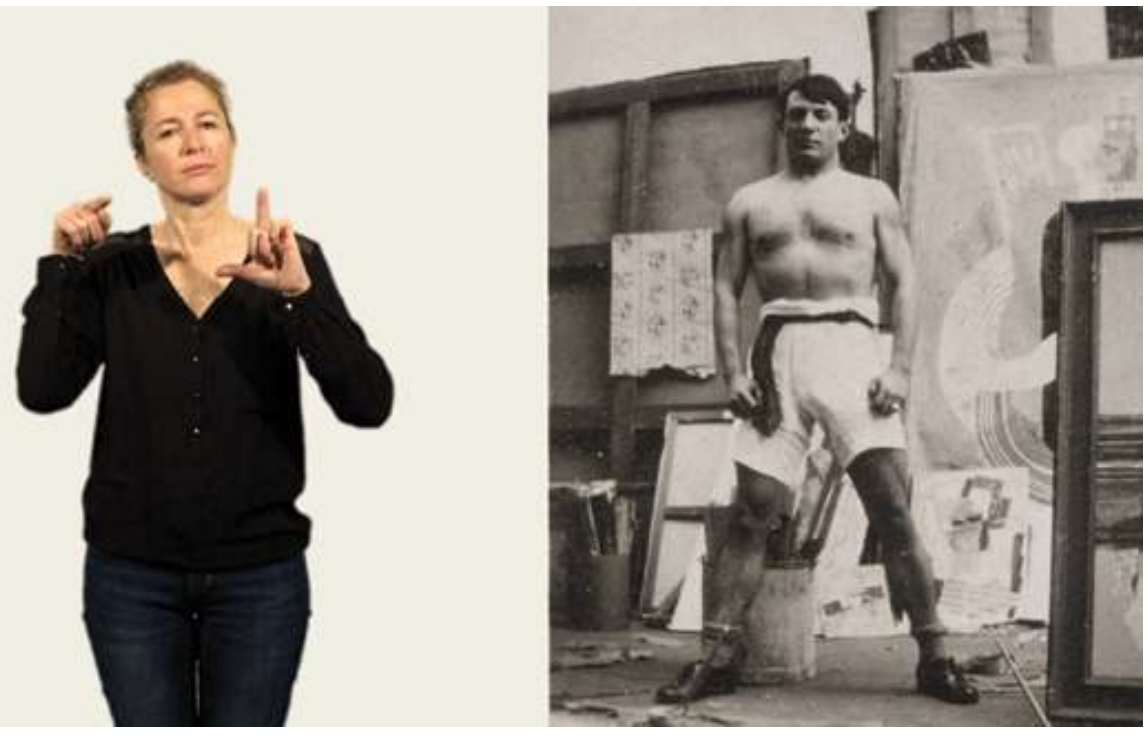

(c) Succession Picasso 2013. Cliché : RMN-Grand Palais/Franck Raux

\section{Les conditions de l'expérimentation}

Quatre dispositifs techniques ont été testés dont trois sont des solutions peu déployées dans les lieux de culture : un visioguide à réalité augmentée, des lunettes et une montre 
connectées. Leur usage par les visiteurs participant a été confronté à celui d'un dispositif abouti et déjà proposé par Audiovisit ${ }^{1}$ au musée national Picasso-Paris : le visioguide classique qui propose des commentaires vidéo en langue des signes française (LSF) ${ }^{2}$. Les participants ont tous utilisé l'ensemble des dispositifs proposés dans le cadre de l'expérimentation : le visioguide classique était proposé sur un appareil ayant un écran de 5,7 pouces tandis que le visioguide en réalité augmentée était proposé sur un écran de 4,2 pouces.

2 Chaque dispositif présentait une œuvre différente, accompagnée du même type de commentaire. Les participants ont d'abord eu en main le visioguide classique, puis la montre connectée, puis le visioguide à réalité augmentée et enfin les lunettes connectées. Ils ont été mis en situation dans les salles (voir encadré page suivante).

3 Au total, dix-sept entretiens (quinze entretiens avec une personne sourde et un entretien avec une personne sourde et une personne entendante) ont été réalisés au format vidéo en LSF et retranscrits par la société spécialisée Mégaphone.

4 L'expression "sourds et malentendants" qualifie deux types de public. Emmanuelle Keruzoré, interprète pour Mégaphone, société de communication en langue des signes basée à Saint-Étienne, précise que les sourds se considèrent comme les représentants d'une langue, la LSF, dont découle une identité culturelle largement revendiquée. Les malentendants, eux, rassemblent les personnes ayant eu peu ou tardivement accès à la LSF et dont la langue première est le français (avec une plus ou moins grande maîtrise).

5 Cette question est directement liée à celle de l'écrit. Lors des entretiens, la demande d'écrit annexé à la LSF émane des personnes dont l'éducation a été essentiellement basée sur la rééducation de la parole et l'accès au français. Aucun des sourds signant, dont l'identité est affirmée clairement dans ce sens, n'a fait cette demande.

6 Le musée national Picasso-Paris a recruté les participants à l'expérimentation en diffusant une annonce auprès d'associations de sourds et malentendants ainsi que de professionnels des musées en charge de l'accessibilité. La grande majorité des personnes ayant participé à l'expérimentation a des pratiques régulières (plusieurs fois par an) ou assidues (plusieurs fois par mois) de visite de musée ${ }^{3}$. La majorité travaille dans le secteur de l'accessibilité, de la culture ou exerce une profession artistique, mais toutes les professions n'ont pas été renseignées au moment de l'entretien. Les participants sont donc plutôt informés et susceptibles de mobiliser leur expertise de la visite culturelle ou de l'accessibilité au cours des entretiens.

7 Dans l'ensemble, les familiers de musées sont aussi utilisateurs des outils et technologies innovantes. Leurs propos renvoient à des expériences vécues dans d'autres contextes et font apparaître une forme d'expertise et de critique mobilisées pour qualifier et juger l'expérience proposée au musée national Picasso-Paris : "À Turin j'ai visité l'Académie des sciences et utilisé des Google glass. Très performant comme technique vraiment..." (Homme, 45 ans, sourd profond, référent LSF dans un établissement pour personnes handicapées, fréquentation : 2-3/an avec un guide signant).

8 L'expérimentation a permis de rendre compte de leur expérience et de leur point de vue sur l'usage de ces dispositifs mobiles lors d'une visite muséale d'un musée d'art pour un visiteur sourd ou malentendant, et tout particulièrement de leur ergonomie (voir encadré ci-contre). Elle a permis d'évaluer la pertinence de l'usage des dispositifs dans le cadre d'une visite culturelle et d'envisager des améliorations ergonomiques touchant 
notamment à la lisibilité des contenus, au rapport à l'information visuelle et écrite à l'écran, au repérage dans l'espace au cours de la visite.

\section{Résultats de l'expérimentation}

Les visiteurs participants ont donné leur appréciation des quatre technologies testées au cours de leur visite en procédant à un classement. Celui-ci place le visioguide à réalité augmentée à la première place, suivi de très près par le visioguide classique, puis par les lunettes et enfin par la montre. Un dernier point permet de rendre compte, de manière transversale, des besoins exprimés par les visiteurs participants, et des pistes de développement qu'ils soulèvent.

Entretien réalisé avec un testeur à la fin de sa participation à l'expérimentation.

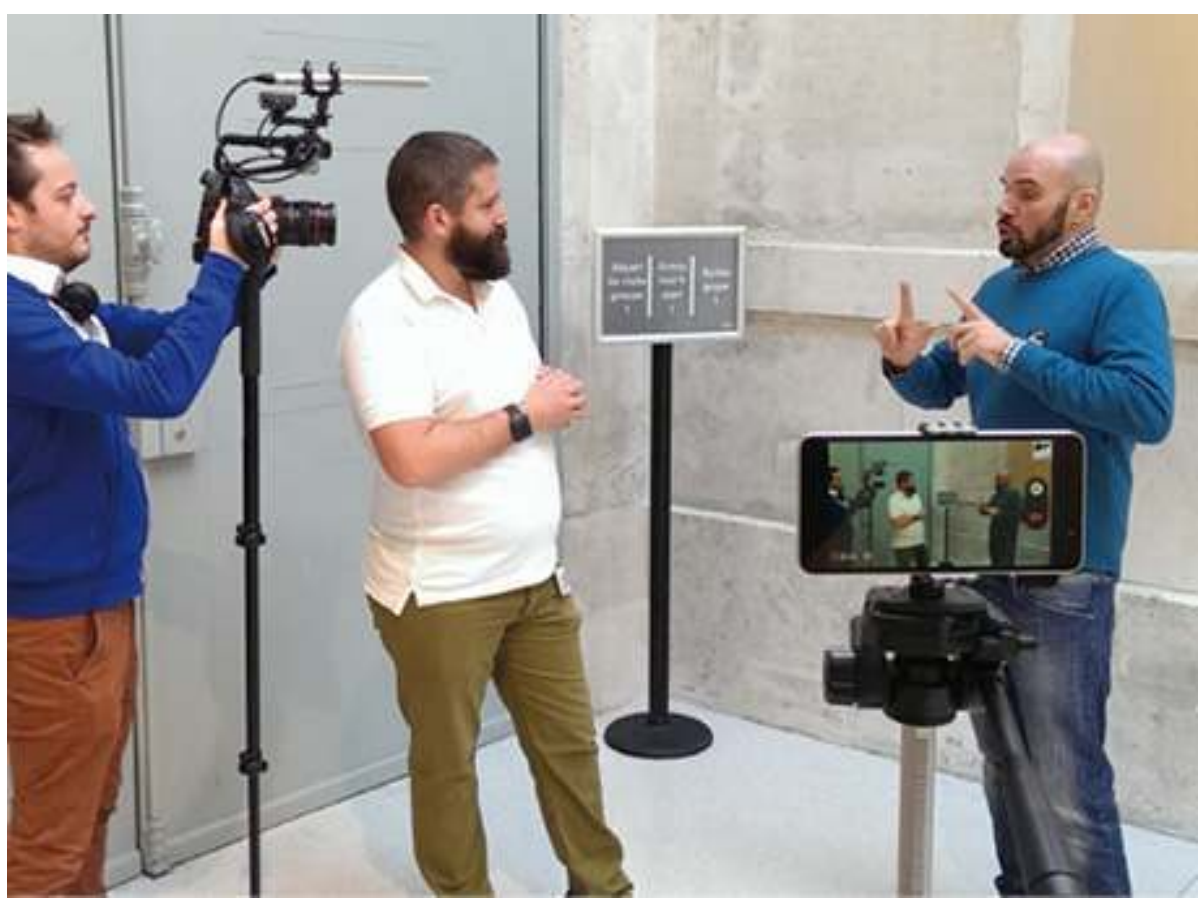

(c) Audiovisit

\section{La relation aux œuvres et la facilité d'usage du visioguide à réalité augmentée plébiscitées}

Le visioguide à réalité augmentée est plébiscité par une grande partie des utilisateurs. Ceux qui y sont le plus favorables lui reconnaissent une "réelle accessibilité" (Femme, 31 ans, sourde, médiatrice culturelle au CMN, fréquentation : 1 à 2/mois). Par accessibilité on peut entendre à la fois : la facilité d'usage pour les visiteurs habitués ou non à utiliser ce genre de dispositif, et la lisibilité du dispositif qui recouvre, du point de vue de visiteurs sourds ou malentendants, une importance particulière.

11 Concernant la facilité d'usage, le dispositif peut ainsi être qualifié de "complètement intuitif", parce qu'"il n'y a pas besoin de réfléchir à comment l'utiliser" (Femme, âge non renseigné, sourde, guide-conférencière Louvre, Orsay, CMN, fréquentation : 30/an). En terme d'ergonomie, le déclenchement automatique du commentaire lorsque le visiteur 
vise l'œuvre est particulièrement apprécié : "Le fait que l'appareil repère seul les œuvres c'est une très bonne chose" (Homme, 45 ans, sourd profond, référent LSF dans un établissement pour personnes handicapées, fréquentation : 2-3/an avec un guide signant).

Le déclenchement automatique est aussi apprécié en raison du rapport aux œuvres qu'il permet. Les participants soulignent l'interaction entre l'œuvre et la "machine" (Homme, 43 ans, sourd profond, belge, professeur de LSF, ancien architecte, fréquentation : 1/mois) ou le "support" proposé par le visioguide à réalité augmentée. Certains le qualifient d'outil qui rend "curieux, donne envie de l'utiliser" (Femme, âge non renseigné, sourde profonde, artiste au chômage, fréquentation : 10/an).

Il faut toutefois noter que certains participants moins habitués des outils numériques d'aide à la visite ont rencontré des difficultés à comprendre la manipulation du dispositif et regrettent l'absence de mode d'emploi. "Il manque une consigne sur sa manipulation. Au départ on tâtonne un peu quant à la position que nous devons adopter, se mettre face à l'œuvre, à quel endroit... Une fois qu'on a trouvé le truc c'est bien" (Homme, 38 ans, sourd, chargé d'accessibilité à la CSI et enseignant à l'université, fréquentation : 2 à 3/ mois).

14 Sur le plan ergonomique, ces derniers ressentent par ailleurs un manque de confort dans la position à adopter qui peut être fatigante (tenir le visioguide à bout de bras, pour le placer entre l'œuvre et soi). Ceux qui ont apprécié le dispositif soulignent sa lisibilité ou sa clarté, ce qui ne les empêche pas de suggérer des modifications pour rendre le dispositif encore plus lisible. Dans ce domaine, la critique la plus récurrente concerne la transparence du fond d'écran lors de la diffusion de commentaires en LSF. Cette transparence perturbe certains participants "il faudrait un fond blanc (derrière l'interprète signeur)" (Femme, 22 ans, sourde, sans emploi, fréquentation : 30/an) ; "j'ai été gênée par la transparence du support" (Homme, 31 ans, sourd, profession non renseignée, fréquentation: 4 à 5/an). Enfin, ajoutons qu'une participante seulement trouve l'écran trop petit : "je préfère les dimensions du visioguide" (Femme, 44 ans, sourde, artiste/infographiste/dessinatrice, fréquentation : 3 à 4/mois).

Les participants les plus favorables identifient également des bénéfices qui touchent plus largement à l'expérience de visite avec ce visioguide. Ils apprécient de se sentir libres de leurs déplacements tout en bénéficiant de commentaires, le visioguide à réalité augmentée apportant ainsi un "vrai confort de visite" (Femme, âge non renseigné, sourde, guide-conférencière Louvre, Orsay, CMN, fréquentation : 30/an). Sa manipulation peut même être perçue comme "ludique" (Femme, 42 ans, sourde, activités diverses, décoratrice, accompagnement d'enfants, cuisinière, fréquentation : 2 à 3/an).

Enfin, un participant souligne explicitement la proximité qu'il perçoit entre le visioguide à réalité augmentée et le visioguide classique, reprochant au premier de ne pas apporter plus que le second (qu'il a placé en première position de son classement). Ce commentaire, et les remarques portant sur la transparence du fond d'écran, incitent à penser que la véritable plus-value du visioguide à réalité augmentée tient davantage au déclenchement automatique par la reconnaissance d'image, à la simplicité d'usage et au rapport aux œuvres associées, qu'à la réalité augmentée en tant que telle. 
Un visiteur sourd teste la visite sur visioguide classique proposée par le musée.

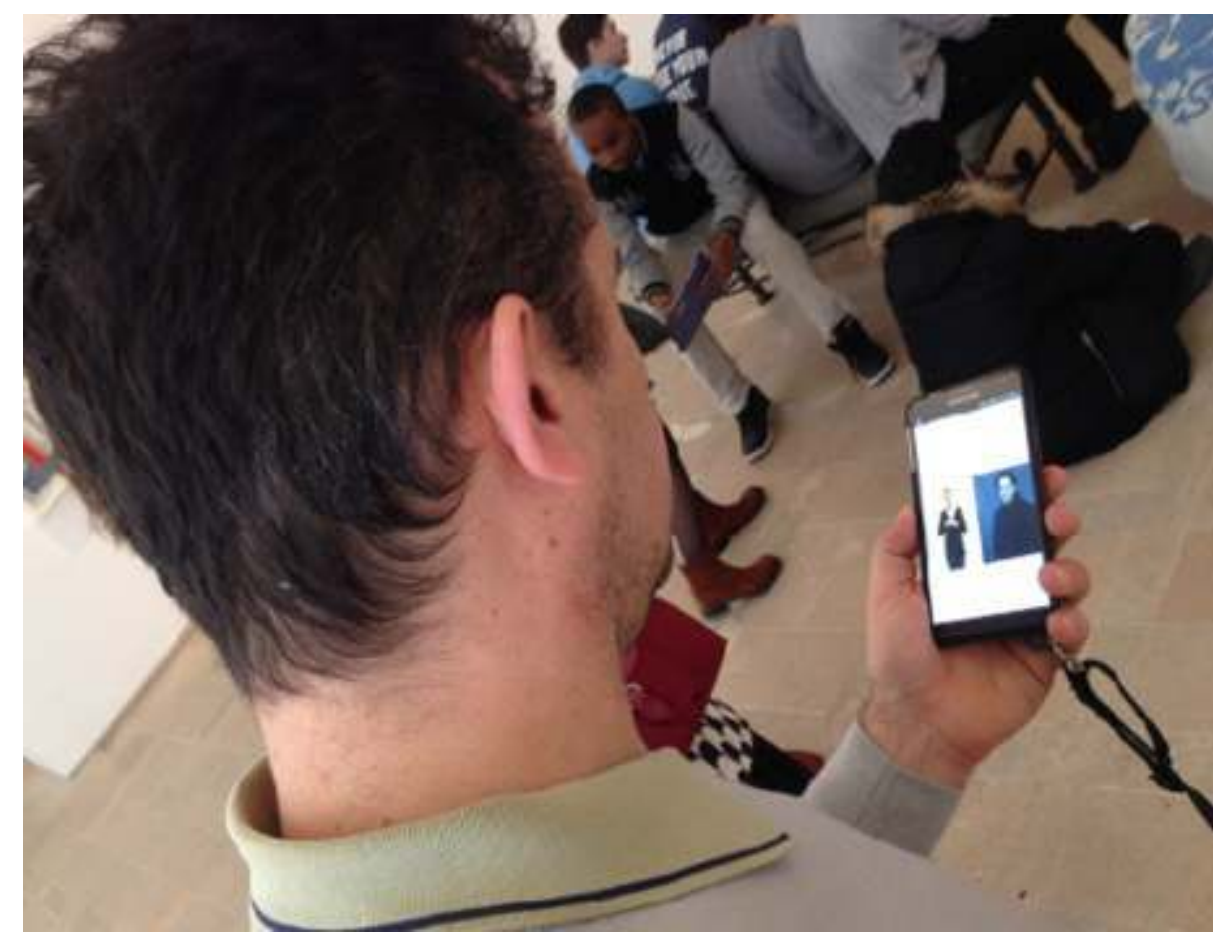

(c) Audiovisit

\section{Le visioguide classique déprécié par une prise en main et une navigation estimées difficiles}

Les commentaires portant sur le visioguide classique sont globalement plus critiques que ceux portant sur celui exploitant la réalité augmentée, y compris de la part des participants qui l'ont le plus apprécié ${ }^{4}$. Le fait que la prise en main du dispositif et la navigation soient difficiles pour beaucoup explique en partie cette différence : "j'avoue que j'étais un peu perdue au début" (Femme, 31 ans, sourde, médiatrice culturelle au CMN, fréquentation : 1 à $2 /$ mois) ; "je n'ai pas tout de suite compris ce qu'il fallait faire" (Homme, 38 ans, sourd, chargé d'accessibilité à la CSI et enseignant à la fac, fréquentation : 2 à $3 /$ mois).

Pour certains, l'incompréhension des icônes du menu accentue cette difficulté : "je ne mettais pas de sens sur les symboles" (Femme, âge non renseigné, sourde profonde, artiste au chômage, fréquentation : 10/an) ; mais c'est surtout l'utilisation du plan et, plus largement le rapport entre l'espace représenté et l'espace parcouru, qui posent problème aux participants. Si l'utilité du plan n'est pas mise en question, celui-ci apparaît difficilement interprétable : "Le plan n'est pas très clair, il est petit, on perd du temps à chercher" (Femme, 38 ans, sourde, technicienne de laboratoire, Toulouse, fréquentation : 10/an) ; "je n'arrivais pas à me repérer, à quoi correspondent tous ces points noirs ?" (Homme, 36 ans, sourd, profession non renseignée, fréquentation : non renseignée).

Lorsqu'elle est mentionnée, la lisibilité du visioguide classique est appréciée. L'image apparait suffisamment grande, agréable à regarder, les signes et les sous-titres sont clairs. Certains mentionnent toutefois le souhait de pouvoir agrandir encore davantage l'image : 
"je voulais zoomer mais il n'y avait pas cette possibilité" (Homme, 45 ans, sourd profond, référent LSF dans un établissement pour personnes handicapées, fréquentation : 2-3/an avec un guide signant).

En ce qui concerne sa manipulation au cours de la visite, si un participant note qu'il peut être fatiguant de l'avoir toujours en main, le dispositif reste peu encombrant, ce qui permet de le glisser dans une poche. Le sens de lecture, dans la longueur ou la largeur de l'écran, n'est pas évident pour certains, qui aimeraient parfois pouvoir changer le sens de lecture, sans modifier les proportions des œuvres représentées.

Enfin, en termes de contenu, si les commentaires sont estimés clairs, il manque à certains des informations écrites. Par ailleurs, la représentation des œuvres commentées à l'écran ne paraît pas utile, voire pertinente, à certains participants. Ils n'envisagent donc pas un usage hors du moment de la visite, face aux œuvres.

Un visiteur sourd teste les lunettes connectées. Le contenu vidéo en LSF s'affiche sur son œil droit. De l'œil gauche, il regarde l'œuvre originale faisant l'objet du commentaire.

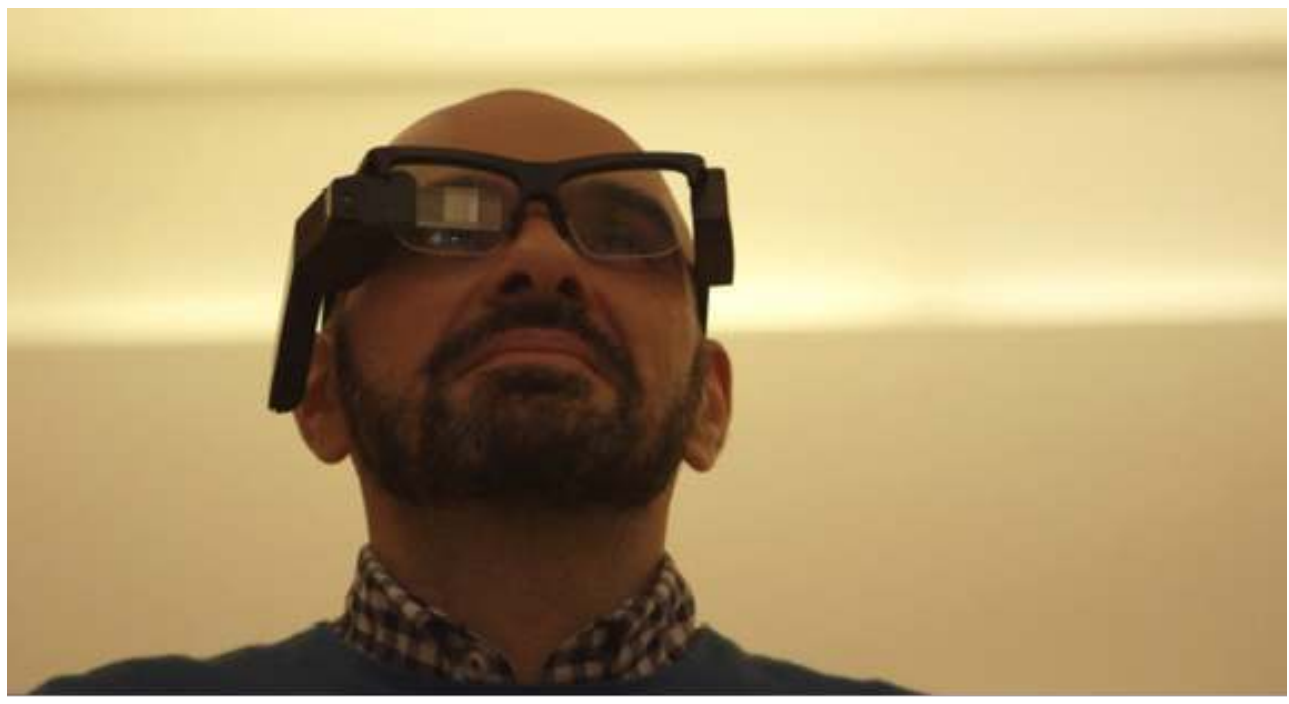

(c) Audiovisit

\section{Les lunettes connectées laissent les mains libres, mais restent à apprivoiser}

Le point commun aux participants qui ont apprécié les lunettes connectées tient au fait qu'elles leur laissent les mains libres : "se déplacer vers une œuvre et avoir juste à presser sur la branche, c'est vraiment bien" (Homme, 45 ans, sourd profond, référent LSF dans un établissement pour personnes handicapées, fréquentation : 2-3/an avec un guide signant).

Malgré cela, l'un d'eux, ayant déjà utilisé des Google glass, estime que la manipulation qui demande de passer son doigt sur la branche n'est pas aisée et fatigante pour le bras (Homme, 38 ans, sourd, chargé d'accessibilité à la CSI et enseignant à la fac, fréquentation : 2 à 3/mois) tandis que d'autres n'apprécient pas l'ergonomie des lunettes qui nécessitent un réglage spécifique en fonction de la taille du visage de l'utilisateur. Certains portant des lunettes de vue estiment, par ailleurs, que les lunettes connectées ne sont pas très adaptées. La manipulation et la relation physique aux lunettes restent donc encore à apprivoiser et fournissent des terrains d'amélioration. 

la fois en raison de la position du corps qu'elle implique, "la position est crispante, elle demande trop d'engagement corporel" (Femme, âge non renseigné, sourde profonde, artiste au chômage, fréquentation : 10/an), et de l'effort visuel demandé en raison de la taille de l'écran : "c'est trop petit, on ne voit pas bien les signes, les mots, c'est fatigant" (Femme, 31 ans, sourde, médiatrice culturelle au CMN, fréquentation : 1 à 2/mois). 
Plus ponctuellement, certains participants signalent qu'il est impossible de signer pendant la consultation de la montre et que le dispositif peut être gênant si l'on porte déjà une montre, faisant ainsi écho au problème soulevé par les lunettes et, plus globalement, à la difficulté d'insertion du dispositif dans les habitudes d'usages.

Un visiteur sourd teste la montre connectée. La vidéo délivre un commentaire en LSF en plein écran. Aucune autre précision n'est apportée à l'écran (iconographie, textes...).

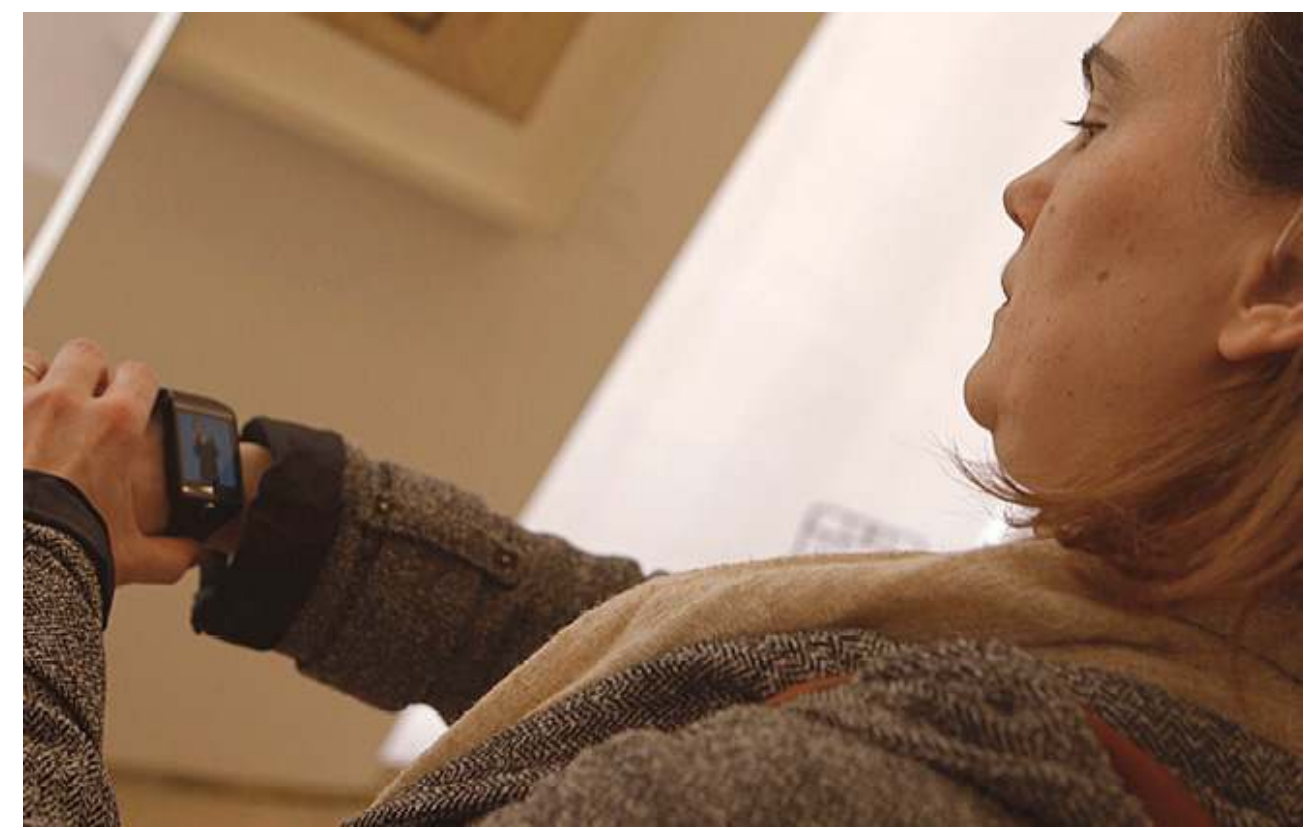

(c) Audiovisit

On peut également noter qu'une des utilisatrices est perturbée par la montre parce qu'elle fait trop directement référence au temps dans sa limite : "Psychologiquement ça me perturbe, j'ai l'impression d'être chronométrée, d'avoir les yeux sur le temps" (Femme, 42 ans, sourde, activités diverses, décoratrice, accompagnement d'enfants, cuisinière, fréquentation : 2 à $3 / a n)$. Certains proposent aussi que celle-ci joue plutôt un rôle d'alerte pour informer, par exemple, de la fermeture prochaine des lieux ou émettre un message de sécurité.

\section{Quelques pistes pour le développement des outils numériques de médiation en LSF}

Les besoins et commentaires exprimés lors de cette expérimentation permettent de dégager quelques pistes concrètes pour le développement des outils numériques de médiation adaptés aux visiteurs sourds et malentendants, et au-delà de souligner les attentes récurrentes propres à ce public.

\section{Améliorer la lisibilité}

Pour les participants, la lisibilité du commentaire en LSF est cruciale, au point qu'ils estiment souvent qu'il n'est pas nécessaire de représenter l'œuvre à l'écran pendant la diffusion du commentaire, à moins de réaliser des gros plans sur l'œuvre. Afficher son 
image au début du commentaire en LSF paraît, en revanche, utile pour le repérage de l'œuvre commentée.

Réaliser des plans plus serrés de la personne signante, permettre de zoomer l'image ou proposer un écran plus grand (le format tablette apparaît peut-être plus adapté à ce besoin, avec le désavantage d'être plus encombrant) pourraient constituer des solutions pour améliorer la lisibilité.

\section{Laisser les mains libres}

Les visiteurs sourds et malentendants parlant la LSF ayant besoin de leurs mains pour signer, cherchent davantage encore que l'ensemble des visiteurs à avoir les mains libres. Les lunettes, du point de vue de la liberté de mouvement, peuvent paraitre la meilleure solution, mais l'adaptation visuelle nécessaire et le contact physique avec les lunettes, ainsi que leur manipulation, disposent d'une certaine marge de progression.

L'un des participants suggère d'accrocher un support sur le ventre, ce qui laisse effectivement les mains libres et permet de proposer un écran plus grand, mais permet difficilement une consultation à plusieurs. Une autre solution envisagée par les participants consiste à disposer des visioguides ou des tablettes fixes près des œuvres, que ce soit des commentaires d'œuvres ou des éléments de contextualisation. Une dernière piste, qui n'a pas été testée lors de l'expérimentation, pourrait être envisagée. Il s'agit de l'utilisation du Google Cardboard ${ }^{5}$. Ce casque de réalité augmentée composé d'une visionneuse en carton dans laquelle est inséré un smartphone diffusant une application adaptée, est plus immersif, mais il coupe son utilisateur de l'environnement immédiat le temps de la consultation.

\section{Permettre de se repérer dans l'espace}

Le repérage dans l'espace est un élément important de l'expérience de visite. Un participant propose une alternative au plan 2D pour se repérer : une vue globale à $360^{\circ}$, ou une vidéo de chaque salle pour mettre en relation espace réel et espace virtuel.

Toutefois, le plébiscite pour le déclenchement par reconnaissance d'image du visioguide à réalité augmentée, qui permet au visiteur de déclencher des contenus de manière automatique, incite à interroger la place du repérage parmi les fonctionnalités intégrées aux outils d'aides à la visite d'exposition. En effet, ce système ne constitue pas un outil d'aide à la localisation, au contraire, il permet de ne pas avoir à se repérer tout en ayant accès aux contenus adéquats. C'est donc finalement une relation directe à l'œuvre, sans passer par l'effort du repérage spatial, qui est souhaitée par les participants.

\section{Proposer plus de choix dans les contenus proposés, et plus de contenus}

Certains participants manifestent le souhait de choisir la forme ou l'approfondissement des commentaires apportés par le dispositif. La possibilité de choisir pour un même contenu entre un commentaire LSF, un texte écrit et un commentaire LSF sous-titré serait appréciée par les visiteurs. D'autres souhaiteraient pouvoir aller plus loin, notamment dans le vocabulaire et les notions techniques, la proposition d'approfondissements 
permettant à chacun de trouver son intérêt. Par ailleurs, le besoin de poser des questions est si prononcé qu'il incite à imaginer des formes de contenu qui permettent de trouver des réponses, au moins pour les questions les plus fréquentes (une forme de FAQ). Enfin, sans être lancée automatiquement, la consigne sur la manipulation pourrait être intégrée à l'ensemble des dispositifs.

\section{Conclusion}

41 À l'issue de ce regard sur l'expérimentation de dispositifs de médiation numérique adaptés au musée national Picasso de Paris, les résultats témoignent de l'expérience que le public sourd a de dispositifs numériques développés dans d'autres institutions patrimoniales comme le Château de Versailles ou la Cité des Sciences et de l'Industrie... ; de l'attrait de la technologie qui "permet au visiteur de faire ses premiers pas vers la recherche de connaissance" (Femme, âge non renseigné, sourde, guide-conférencière Louvre, Orsay, CMN, fréquentation : 30/an) ; et de l'importance de l'accès à l'information pour tous : "Les sourds ont besoin de se forger une culture à l'égal des entendants (...) c'est injuste de ne pas avoir accès aux mêmes informations, on se sent mis de côté" (Homme, 45 ans, sourd profond, référent LSF dans un établissement pour personnes handicapées, fréquentation: 2-3/an avec un guide signant). Cela incite à développer l'offre de médiation, y compris des applications téléchargeables sur les smartphones des utilisateurs, associées à des liens vers des ressources culturelles adaptées.

Ils montrent aussi que l'interaction humaine est souhaitée par les visiteurs : "moi j'aime la technologie humaine. C'est-à-dire, un vrai guide conférencier" (Femme, 22 ans, sourde, sans emploi, fréquentation: 30/an) ; mais qu'en l'absence de visite guidée en LSF, les dispositifs individuels sont attendus parce qu'ils apportent une grande liberté et une vraie alternative.

Entre autonomie et interaction, il convient donc de veiller à ce que le dispositif technique ne se substitue pas à la médiation humaine. "Les deux méthodes se complètent (...) J'aime cette autonomie que me donne l'audioguide. Mais j'aime aussi faire une visite avec un guide qui me donnera plus de contexte, des explications différentes. Et puis être dans l'interaction. (...) Il ne faut pas que la technique remplace l'humain" (Femme, âge non renseigné, entendante, parle la LSF, employée à l'accessibilité tout public des expositions de la CSI, fréquentation : $1 /$ sem).

Le musée apparait, par ailleurs, aux yeux des participants, comme un lieu pertinent pour l'expérimentation d'outils de médiation, en l'occurrence d'outils adaptés. Elle soulève d'ailleurs des problématiques qui rejoignent celles auxquelles les publics sont confrontés face au numérique de manière générale, en termes d'ergonomie (mode d'emploi, compréhension des symboles, lisibilité, réactivité), d'aide à l'orientation dans l'espace, ou de navigation dans les contenus. Certains participants le relèvent d'ailleurs : "je pense même que c'est un outil (le visioguide à réalité augmentée) tout à fait adapté à des personnes âgées, des personnes à la retraite, peu réceptives à la technologie. Ça apporte un vrai confort de visite" (Femme, âge non renseigné, sourde, guide-conférencière Louvre, Orsay, CMN, fréquentation : 30/an). Cela rejoint la thèse d'une accessibilité pour tous d'outils de médiation adaptés répondant à des besoins spécifiques comme en témoignent d'autres études ${ }^{6}$. 


\section{Le dispositif d'expérimentation}

L'expérimentation s'est déroulée au musée national Picasso-Paris. Les œuvres commentées ont été choisies par Audiovisit de manière à provoquer le déplacement des testeurs dans les salles, et notamment leur passage du rez-de-chaussée au 1er étage, afin de les mettre en situation de visite.

Pour les besoins de l'expérimentation, des contenus qui avaient déjà été développés pour le visioguide classique en LSF ont été réemployés. Le visioguide classique, testé en premier, comportait une introduction de 1 minutes et 17 secondes présentant à la fois le musée national Picasso-Paris et le principe d'utilisation du visioguide. Toujours au rez-dechaussée, la montre proposait un commentaire de 2 minutes et 20 secondes sur le buste en bronze baptisé Le Fou daté de 1905. Les testeurs se rendaient ensuite au 1er étage pour expérimenter le visioguide en réalité augmentée à travers un commentaire de 4 minutes et 14 secondes sur le Portrait d'olga dans un fauteuil. Cette huile sur toile, réalisée à partir d'une photographie, est datée de 1918. Enfin, les lunettes ont été testées au 1er étage à partir d'un commentaire de 2 minutes et 47 secondes du Portrait de Dora Maar portrait cubiste daté de 1937.

La grille d'entretien était organisée de la manière suivante :

- Quel âge avez-vous?

- Êtes-vous malentendant, signeur, sourd?

- Travaillez-vous pour un musée ? Si non, combien de fois par an allez-vous au musée?

- Avez-vous l'habitude de faire des visites de musée sans guide ? Des visites avec un guide en LSF ? D'utiliser des visioguides au musée?

- Qu'avez-vous pensé du visioguide, du visioguide à réalité augmentée, des lunettes connectées, de la montre connectée ? De la machine utilisée ? De l'image à l'écran ? De la facilité d'utilisation? Des explications données?

- Qu'est-ce que vous avez aimé ? Qu'est-ce que vous n'avez pas aimé ?

- Est-ce que vous imaginez visiter tout le musée avec ce visioguide, ce visioguide à réalité augmentée, ces lunettes connectées, cette montre connectée?

- Avez-vous des améliorations à proposer?

\section{BIBLIOGRAPHIE}

Bertin, F. Les sourds, une minorité invisible. Paris : Éditions Autrement, 2010.

Bertin, F. Les sourds, l'affirmation d'une identité, Sciences humaines, n²53, 2013, pp. 52-57.

Goodwin, H. American sign language and audio description on the mobile guide at the museum of fine arts, Boston, Curator : The Museum Journal. Vol. 56, Issue 3, July 2013, pp. 369-370.

ICOM. Dossier : Des musées (plus) accessibles, La vie des musées, n²4, 2012, pp. 9-147. 
Signes de sens, DeVisu, Geriico, ScaLab. Design for all et contenus culturels : évaluation de dispositifs de médiation visuelle pour tous. Rapport d'évaluation du projet mené dans le cadre du programme "Chercheurs \& Citoyens" 2013-2015. www.signesdesens.org/wp-content/ uploads/2015/11/2015_11_Evaluation-design-for-all-PCC_Diff.pdf

Zedda, M., Hearn, K., Bowen, J.-P. and Linsey, E. Museum and technology : being inclusive helps accessibility for all, Curator: The Museum Journal. Vol. 56, Issue 3, July 2013, pp. 353-361.

\section{NOTES}

1. Audiovisit est concepteur et fournisseur de solutions de visites embarquées dans les musées, lieux d'exposition, monuments et villes depuis 2002 avec plus de 300 projets de visite à son actif.

2. En 1999, 9 \% des Français sourds ou malentendants déclaraient utiliser la langue des signes française : www.2-as.org/site/IFSI-2011/1-3-Statistiques-INSEE-1998-1999.pdf

3. Les grandes enquêtes nationales, comme celles du Crédoc (2012) www.credoc.fr/pdf/Rapp/ R281.pdf ou Les Pratiques culturelles des Français (2008). www.pratiquesculturelles.culture.gouv.fr/doc/tableau/chap7/VII-3-2-Q75B.pdf dénombrent les visites et leur fréquence, mais ne qualifient pas les usages. Les catégories de pratiques "régulières" ou "assidues" sont donc élaborées dans le cadre de cette expérimentation et ne sont pas comparables à des catégories préexistantes.

4. Suite à l'expérimentation, le visioguide classique du musée national Picasso-Paris a été modifié, cette expérimentation ayant aussi comme objectif d'évaluer l'offre de visioguide mise en place quelques semaines auparavant au musée. Ainsi, l'affichage des vidéos en LSF en mode plein écran a, par exemple, été amélioré.

5. Voir en ligne : https://vr.google.com/cardboard.

6. Voir par exemple : Comment la rencontre d'un public déficient visuel éclaire les attentes du public ordinaire, in Chauvey, V. Les publics déficients visuels au muséum de Lyon. Université Grenoble 2, 2007

\section{RÉSUMÉS}

L'expérimentation menée au musée national Picasso-Paris vise à évaluer le potentiel de plusieurs dispositifs numériques mobiles en tant qu'outils de médiation pour les visiteurs sourds et malentendants. Les entretiens réalisés suite aux tests permettent de dégager des pistes pour le développement de tels dispositifs susceptibles de répondre aux besoins de ce public spécifique.

\section{INDEX}

Mots-clés : ergonomie, dispositif numérique, accessibilité, public 


\section{AUTEURS}

\section{GAËLLE LESAFFRE}

directrice d'études "Publics, Culture \& Numérique"

lesaffregaelle@gmail.com

\section{GUILLAUME DUCONGÉ}

directeur exécutif de la société Audiovisit

g.duconge@audiovisit.com 